\title{
Impact force magnitude analysis of an impact pendulum suspended in a vibrating structure
}

\author{
R.R. Aguiar* and H.I. Weber \\ Department of Mechanical Engineering, Pontifical Catholic University of Rio de Janeiro, Rio de Janeiro, Brazil
}

Received 14 October 2011

Revised 30 January 2012

\begin{abstract}
The purpose of this work is the experimental investigation and the mathematical modeling of the impact force behavior in a vibro-impact system, where an impact pendulum is mounted on a cart that moves with a prescribed displacement. The dynamics of the system will be evaluated considering different excitation frequencies and changing the impact gap. Experimental data are used to validate the mathematical model. The mathematical model allows a detailed nonlinear analysis, showing the rich response of the system, which includes dynamical jumps, bifurcations and chaos. In impact systems, discrepancies between numerical results and experimental measurements are common due to the difficulty in describing all factors that influence the resulting impact force profile. The use of wires to suspend the impacting body has the purpose to limit these uncertainties.
\end{abstract}

Keywords: Nonlinear dynamics, impact force, vibro-impact, impact oscillator

\section{Introduction}

Oil well drilling in hard rock formations is still a great challenge for oil companies. Optimum productivity is possible by combining advantages of both existing drilling techniques: rotary and percussive drilling. In conventional rotary drilling, the energy applied in the system (oil well drillstring) comes from the rotary table, located at the top of the drillstring. Such energy, supplied to drill the oil well, ends up being wasted in vibration (axial, torsional and lateral), friction with borehole walls or casing and heat [1-3]. If part of the energy wasted in vibration could be reinserted into the drilling process, the rate of penetration (ROP) can be increased.

The motivation of this work is to use the already existent vibrations in the drillstring [2,4], to generate an harmonic load on the bit and an excitation in a steel mass (hammer) which will cause impacts, see Fig. 1 . The concept of this hybrid drilling technique is to reinsert the energy wasted on axial vibration, back into the drilling process, with the use of impacts. The stress waves created by such impacts may be useful to release the system from a stick condition of stick-slip phenomena, as well as generating cracks on the rock formation (in case of brittle lithologies), increasing the rate of penetration. The axial vibration generated by the bit/rock interaction excites a hammer. When the excitation frequency approaches the mass resonance, impacts on the bit occur, because the hammer displacement is limited by the gap. Therefore, in addition to the rotary drilling, a percussive action happens due to the impact of the hammer on the bit $[5,6]$. The idea of combinining a percussive action to rotary drilling is not new, being first developed by Hausser and Nüsse \& Gräfer in 1955, as referenced in the works of Batako et al. [5].

The study of vibro-impact systems has been the aim of several researches, from the application of a percussive action in rotary drilling for improved performance [5-7], to ultrasonic drilling [8-10], impact dampers [11,12] and vibro-safe percussion machines [13].

\footnotetext{
* Corresponding author: R.R. Aguiar, Department of Mechanical Engineering, Pontifical Catholic University of Rio de Janeiro, Rio de Janeiro, Brazil. E-mail: aguiar.rr@gmail.com.
} 


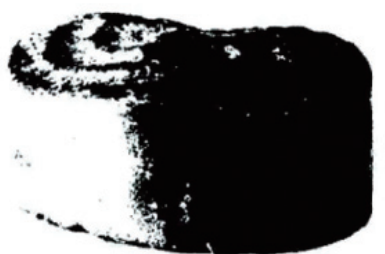

(a)

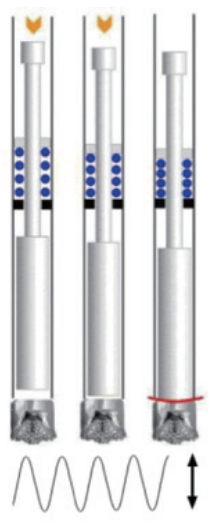

(b)

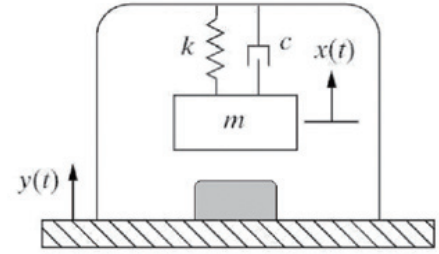

(c)

Fig. 1. a) Trilobe formation on hard rocks caused by roller cone bits drilling; b) Resonance hammer drilling technique concept; c) Embedded vibro-impact system.

In general, experimental contributions have been rather limited. Shaw and Holmes [14] experimentally examined the response of a beam with a fixed amplitude constraint at one end, noting that a one degree of freedom approximation allowed prediction of the regions of periodic and chaotic motion. This was extended systematically in Shaw [15], where the subharmonic resonances predicted in Thompson et al. [16] were observed along with period-doubling bifurcations. Ing et al. [17] also studied an impact oscillator with a one-sided elastic constraint. Different bifurcation scenarios have been shown for a number of values of the excitation amplitude, with the excitation frequency as the bifurcation parameter. Mann et al. [18] investigates the application of a finite time impact model to the study of a parametrically excited planar pendulum subjected to a motion-dependent discontinuity. Such studies demonstrate the presence of multiple periodic attractors, subharmonics, quasi-periodic motions, and chaotic oscillations. Various other experimental impact oscillators were studied by Hinrichs et al. [19] and Todd and Virgin [20]. Of note is Piiroinen et al. [21], where a pendulum contacting with a rigid stop was shown to exhibit periodic windows in a period adding cascade up to period-5.

From the theoretic point of view, vibro-impact systems are a quite interesting subject [22], because it presents a rich and complex dynamical response, from periodic to chaotic behavior. Since it is a nonsmooth system, bifurcations and other nonlinear phenomena may occur, where such behaviors are mostly not present in linear systems. Besides the references mentioned above, several other studies concerning vibro-impact systems are available in the literature, such as the works of Savi et al. [23,24], and Peterka [12,25-27].

Considering the available literature, authors decided to investigate a simplified hammer structure, impacting on board of a vibrating structure and looking for the conditions of maximum impact force. The experiment should be modeled and validated. The results using an elastic supported hammer presented an interesting amount of different nonlinearities (Aguiar et al. [28]), but included discrepancies in the validation due to hammer dynamics. This lead to the present paper where the experiment consists in a hammer supported by 8 strings impacting on a load cell, all embedded in a cart performing a harmonic motion with controlled amplitude and frequency. The objective is to understand the system dynamics demanded for a shorter time scale analysis in the contact process and a mathematical model which, validated, will be able to extend the range of analysis. The main result will be summarized in a map that presents the impact regions associated to the maximum impact force and is a variation of map showing the regions of stable periodic behavior (called by the authors 'Peterka Map').

Although the literature shows several studies dealing with vibro-impact systems, as listed, there is few concern regarding the force magnitude developed by the impacts. Since the motivation relies on a hybrid technique to drill hard rock formations, special attention is dedicated in this work regarding the impact force magnitude, as well the parameters combination thats generates higher impact forces. The authors propose a contribution to the field of vibro-impact dynamics proposing the Peterka map with impact force magnitude addressed as a design tool for this special type of devices. 


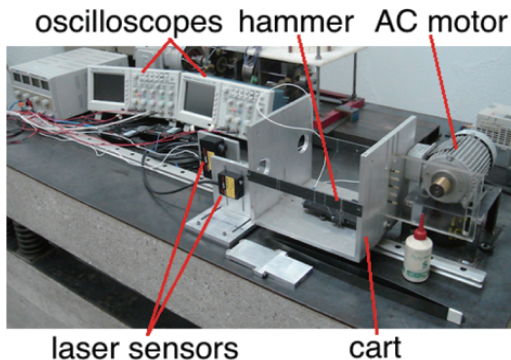

(a)

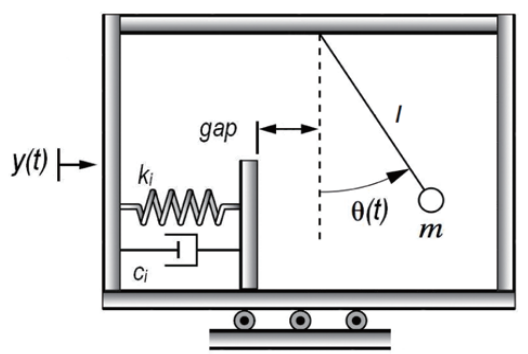

(b)

Fig. 2. Test rig: a) Picture of the entire test rig, including acquisition hardware; b) Physical representation.

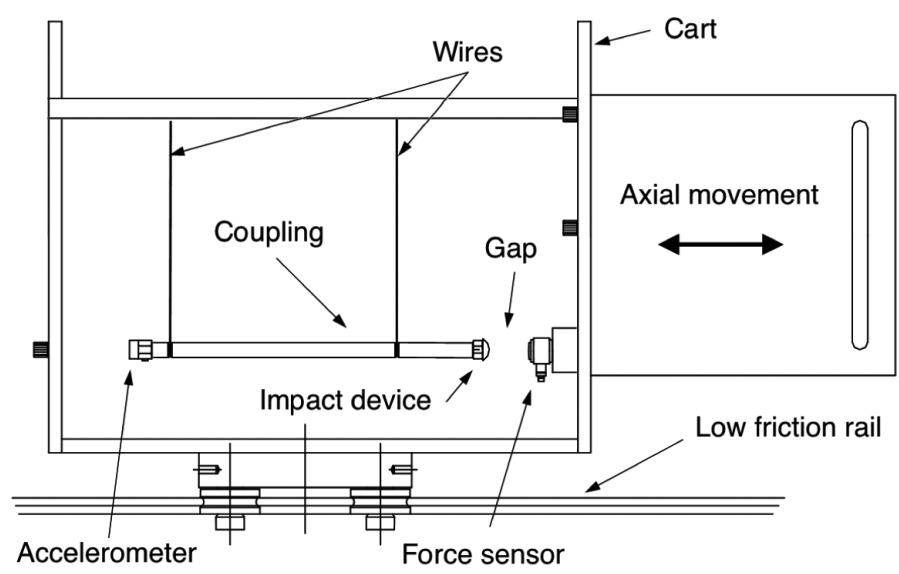

Fig. 3. Test rig: Cart and hammer sketch.

\section{Experimental apparatus}

The experimental apparatus is shown in Fig. 2. The experiment consists of a main cart, made of aluminum, which slides along the horizontal axis on a low friction rail bearing assembly, see Fig. 3. The main cart is excited by an inverter controlled AC motor. The motor is attached to the cart through a pin that slides into a slot machined on an acrylic plate attached to the cart. The pin hole is drilled off-centered on the disk at the edge of the motor, so that rotational motor movement becomes sinusoidal cart movement. This device is used instead of an electromagnetic shaker because it can perform higher hammer amplitudes than a shaker. The device also avoids the influence of impact forces on the excitation source at higher frequencies.

The hammer is fixed inside the main cart, Fig. 2(a), its weight supported by a wires set up. The entire system moves in a horizontal axis. As shown in Fig. 2(a), eight wires are used, assuring that the hammer moves in the same axis as the main cart. The wires are attached to the cart structure at an angle of 30 degrees approximately from the vertical axis, minimizing the hammer rotation after impact. The hammer is composed of aluminum with a steel impact device. To vary the gap between the hammer and the cart, the impact device is composed of a screw and a knurled nut. The measurement devices on the test rig include (see Table 1):

- one accelerometer attached to the hammer;

- one piezoelectric force sensor fixed to the cart and located in front of the hammer impact device;

- two laser displacement sensors, both located on the side of the cart. One of the laser displacement sensors measures cart displacement and the other measures hammer displacement.

All data is acquired by two oscilloscopes using different time scales. The first oscilloscope measures the impact force and acceleration at the precise moment of impact (micro scale), after the impact force signal is triggered. The second oscilloscope measures both cart and hammer displacements using laser displacement sensors signals (macro scale). 
Table 1

Sensor specs

\begin{tabular}{lcc}
\hline Cart accelerometer - 751-10 SN AC69 & \\
Sensitivity & 10.194 & $\mathrm{mV} / \mathrm{g}$ \\
Measure Range & \pm 50 & $\mathrm{~g}$ \\
Resonance frequency & 50 & $\mathrm{kHz}$ \\
Impact force sensor - 2311-100 SN 2471 & & \\
Sensitivity & 24.41 & $\mathrm{mV} / \mathrm{N}$ \\
Measure Range & \pm 220 & $\mathrm{~N}$ \\
Resonance frequency & 75 & $\mathrm{kHz}$ \\
Cart laser displacement sensor - optoNCDT 1607-20 & & \\
Sensitivity & 10 & $\mathrm{~V} / \mathrm{mm}$ \\
Measure Range & 20 & $\mathrm{~mm}$ \\
Hammer laser displacement sensor - optoNCDT 1607-100 & & \\
Sensitivity & 2 & $\mathrm{~V} / \mathrm{mm}$ \\
Measure Range & 100 & $\mathrm{~mm}$ \\
\hline
\end{tabular}

\subsection{Experimental methodology}

The test rig considers different values for the impact gap and the excitation frequency. The length of the gap is measured using a calibrated shim. The excitation frequency is supplied by the AC motor. The system responses are: the hammer acceleration; the impact force; the cart displacement and the hammer displacement.

The methodology is to observe the impact force behavior as the gap is varied. First, the natural frequency of the system without impact is determined, as well as the system parameters. After that, a study with impact is carried out. The excitation frequency is varied in order to cover the possible range of excitation frequencies. Three different gap values were chosen: $0.0 \mathrm{~mm}, 1.0 \mathrm{~mm}$ and $2.4 \mathrm{~mm}$. In all results shown below the data was acquired after the transient response.

The laser displacement sensor signal presents an undesirable level of noise, which was removed using a moving average filter. Due to the nature of the moving average (a low-pass filter) the phase plane charts show a smooth effect during the impact, which is an effect of the differentiation of low-pass filtered signal, and does not reflect the reality of the impact, as will be seen during the comparison between experimental data and numerical results.

\section{Experimental results}

If there is no impact, the hammer behaves as a one-degree-of-freedom system excited by a harmonic load (in this case, a base excitation). Since such system is well known and widely described in literature [30], no further comments are required. For each acquisition, all data was collected after a considerable amount of time in order to remove the transient behavior from the response.

\subsection{Experimental results for gap $0.0 \mathrm{~mm}$}

Hammer impact force behavior can be split into frequency bands, showing similar characteristics in each frequency band for all gaps. In a low excitation frequency range (less than $2.5 \mathrm{~Hz}$ ), the cart movement is so slow that the hammer basically follows the prescribed excitation, generating two or three impacts per excitation cycle with low force magnitude. Due to the low impact force magnitude produced, such frequency range is not discussed in this work. In the next level of frequencies, the impact force presents a period-1 $(z=1 / 1,1$ impact per 1 excitation cycle) stable behavior. The impact force magnitude increases as the excitation frequency increases, reaching its highest value at $3.75 \mathrm{~Hz}(82.4 \mathrm{~N})$ and after this frequency the impact force decreases as the frequency is raised. Figure 4 shows the impact force, hammer acceleration and both cart and hammer absolute displacements for the maximum impact force of frequency band $z=1 / 1$.

Regarding the impact force measurements, the impact force transducer captures the first impulse transferred by the hammer, reaching its maximum. After the first impulse, in the micro scale time analysis, the support structure 


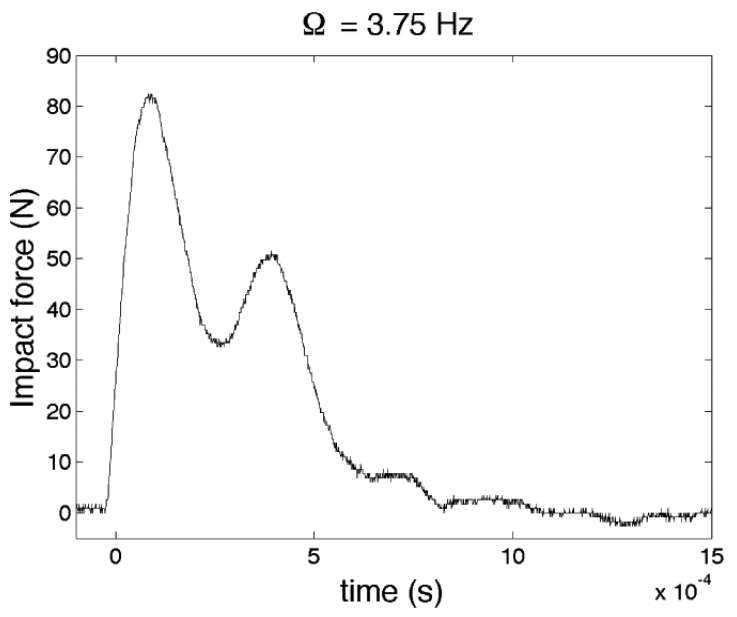

(a)

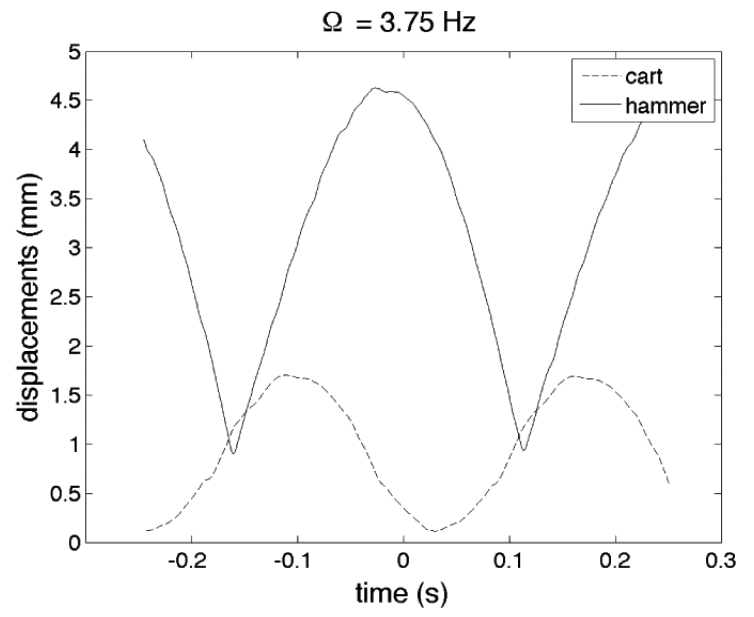

(b)

Fig. 4. Gap 0.0 mm. Excitation frequency $3.75 \mathrm{~Hz}$ : a) Impact force over time; b) Displacements (cart and hammer).

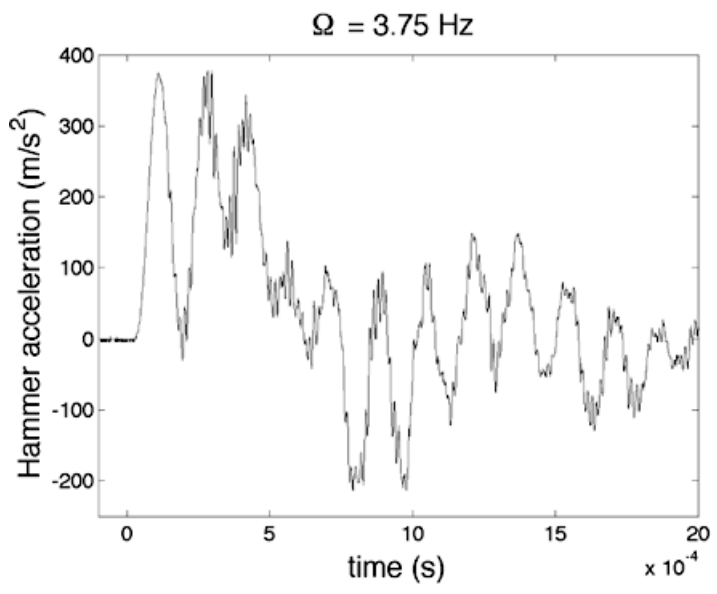

(a)

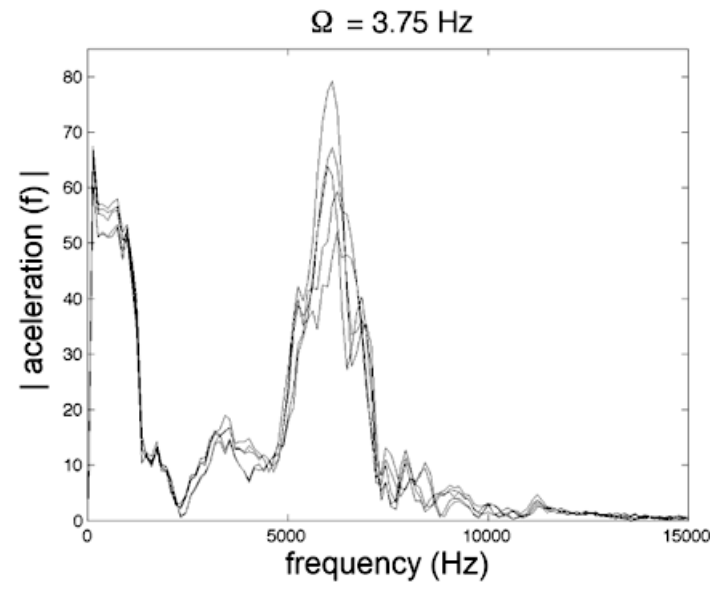

(b)

Fig. 5. a) Gap $0.0 \mathrm{~mm}$, excitation frequency $3.75 \mathrm{~Hz}$ : Hammer acceleration during impact; b) FFT of hammer acceleration signal after several impacts.

bounces back transferring energy to the hammer. The contact dynamics reacts according to its own dynamics, reaching a second peak, see Fig. 4(a). The accelerometer measures the hammer dynamics, because it is fixed to the opposite side of the impact device, see Figs 2(a) and 3. The existence of contact dynamics is strengthened by the results shown in the acceleration chart, Fig. 5(a), because there are unexpected oscillations after the impact. Figure 5 shows the FFT of several acceleration signals taken after each impact. From the charts 3 peaks are observed. The first one is related to the pendulum natural frequency $(1.82 \mathrm{~Hz})$. The second peak $(3 \mathrm{kHz})$ is related to the bending flexibility of the cart plate where the impact force sensor is mounted. The last peak occurs at around $6 \mathrm{kHz}$, and corresponds to the hammer axial vibration.

At the end of frequency band $z=1 / 1$, the hammer undergoes a bifurcation in the impact force behavior under excitation frequency variation, characterized by a period-1 impact with low magnitudes at alternate impact magnitudes, as shown in Fig. 6.

The second frequency band presents a similar behavior, described above. In this frequency band the impact force occurs every two excitation cycles $(z=1 / 2$, one impact every two excitation cycles), with the impact force peak 


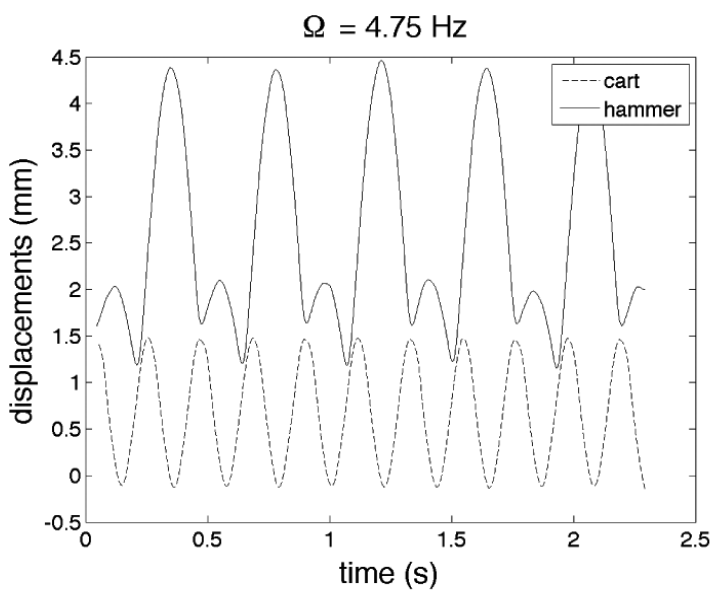

(a)

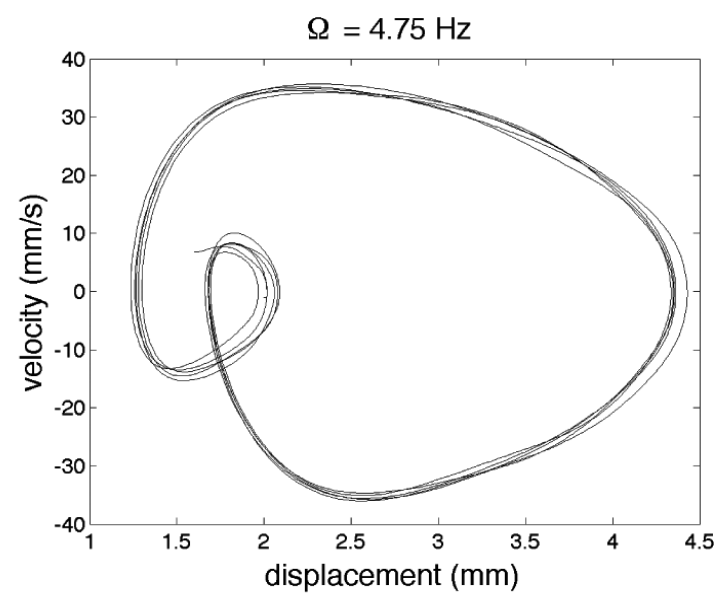

(b)

Fig. 6. Gap $0.0 \mathrm{~mm}$. System undergoes a bifurcation. Excitation frequency $4.75 \mathrm{~Hz}$ : a) Displacements; b) Hammer phase plane.

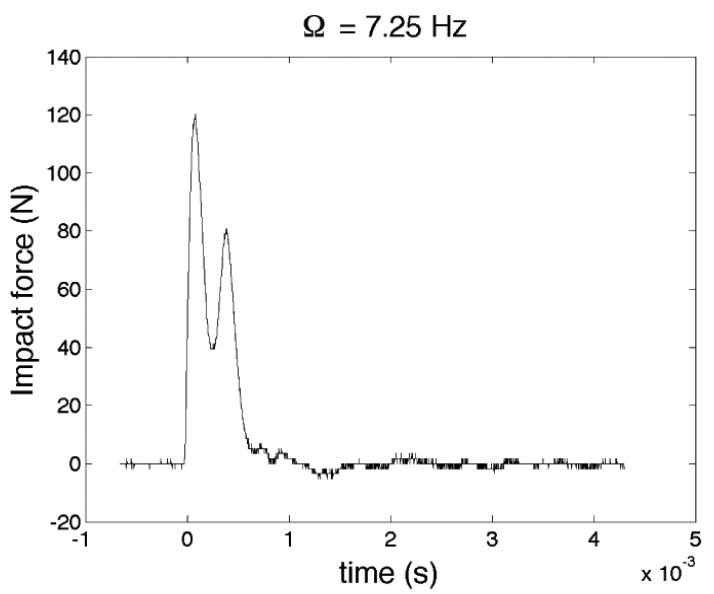

(a)

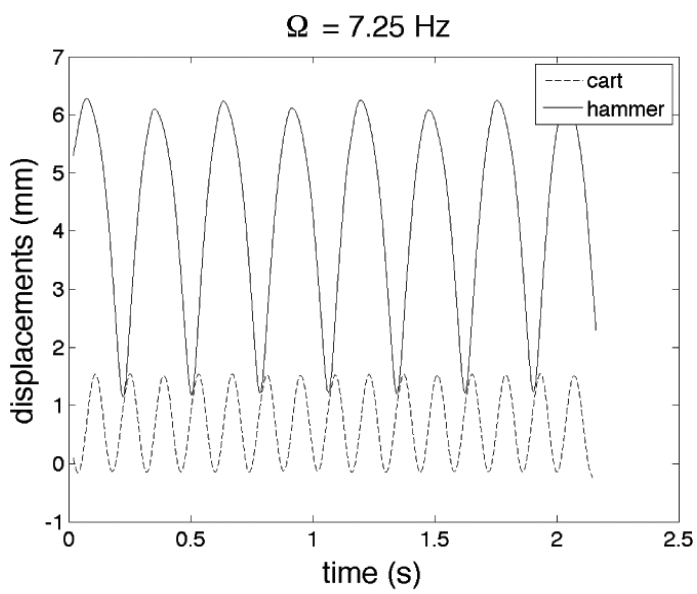

(b)

Fig. 7. Gap $0.0 \mathrm{~mm}$. Maximum impact force on frequency band $z=1 / 2$. Excitation frequency 7.25 Hz: a) Impact force; b) Displacements.

increasing as the frequency increases, reaching its highest value at $7.25 \mathrm{~Hz}(120 \mathrm{~N})$, see Fig. 7. The excitation frequency where the maximum impact force occurs on this frequency band $(z=1 / 2)$ is twice that of frequency band $z=1 / 1$. After the activity at frequency band $z=1 / 2$ the system undergoes another bifurcation, see Fig. 8 .

After each period-doubling bifurcation, a nonperiodic behavior is observed when increasing the excitation frequency, as shown in Fig. 9. The Lyapunov exponents were not calculated in order to confirm if the nonperiodic behavior is chaotic.

With this experimental data it is possible to analyze the system behavior in the frequency domain. To do so, a computational routine has been developed to determine the $F_{i}$ (impact force peak). The maximum value of $F_{i}$ has been extracted for each excitation frequency. Since the impact force peak does not change within each excitation frequency, except for the behavior after bifurcation, this routine seems to be quite effective. Finally, to generate a non-dimensional chart the force ratio $F_{i} / m g$ is used ( $m g$ is the hammer weight), and the excitation frequency is divided by the natural frequency of the hammer without impact. The natural frequency of the hammer is experimentally identified using modal analysis, see Table 2. This non-dimensional chart is useful to compare data between different hammer configurations. 
Table 2

\begin{tabular}{lcc}
\multicolumn{3}{c}{ Impact resonance frequencies (experimental) } \\
\hline Frequency band & $\begin{array}{c}z=1 / 1 \\
(1 \text { impact/ cycle })\end{array}$ & $\begin{array}{c}z=1 / 2 \\
(1 \text { impact/ } 2 \text { cycles })\end{array}$ \\
\hline gap $0.0 \mathrm{~mm}$ & $3.75 \mathrm{~Hz}$ & $7.25 \mathrm{~Hz}$ \\
gap $1.0 \mathrm{~mm}$ & $3.00 \mathrm{~Hz}$ & $6.25 \mathrm{~Hz}$ \\
gap $2.4 \mathrm{~mm}$ & $2.50 \mathrm{~Hz}$ & $5.25 \mathrm{~Hz}$ \\
System natural frequency $(\mathrm{gap} \rightarrow \infty)$ & $1.82 \mathrm{~Hz}$ & \\
\hline
\end{tabular}

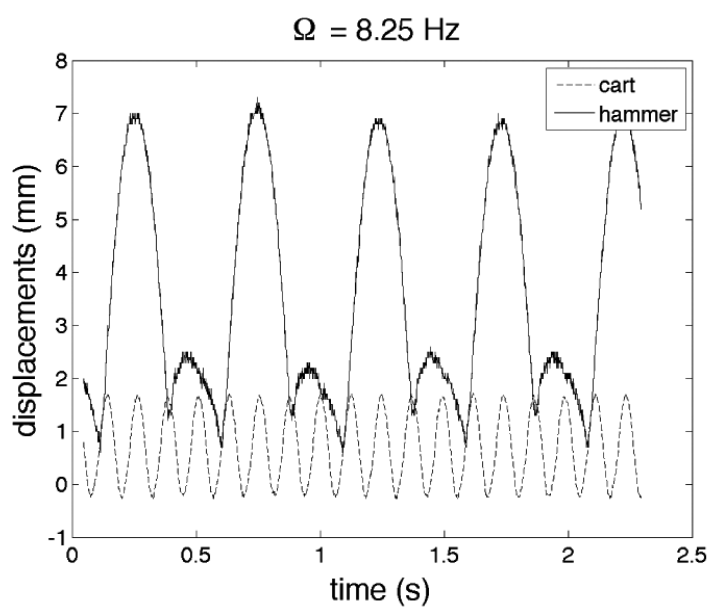

(a)

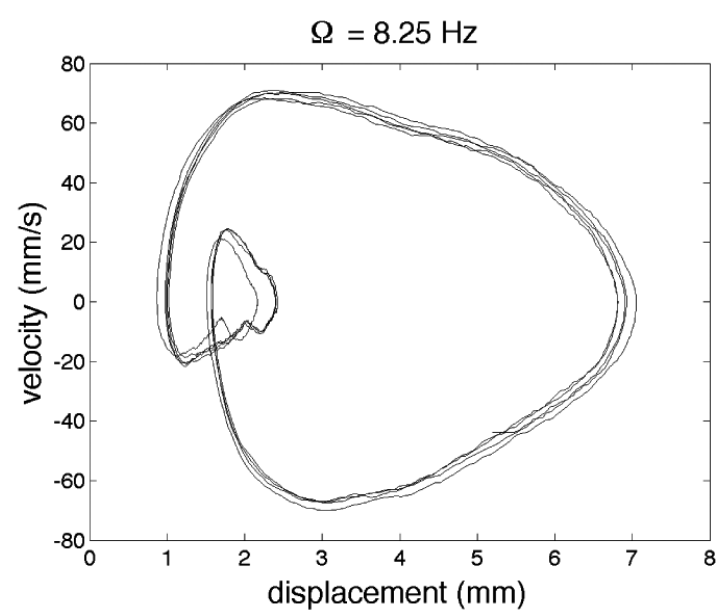

(b)

Fig. 8. Gap 0.0 mm. System undergoes a bifurcation. Excitation frequency $8.25 \mathrm{~Hz}$ : a) Displacements; b) Hammer phase plane.

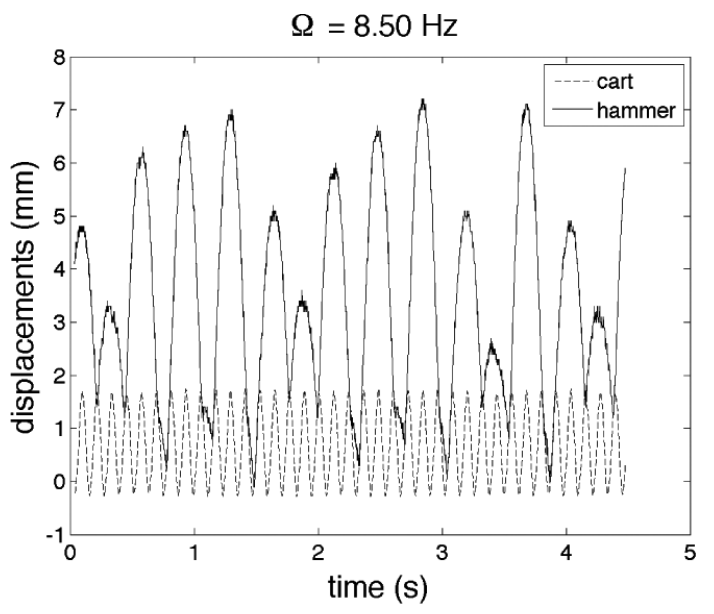

(a)

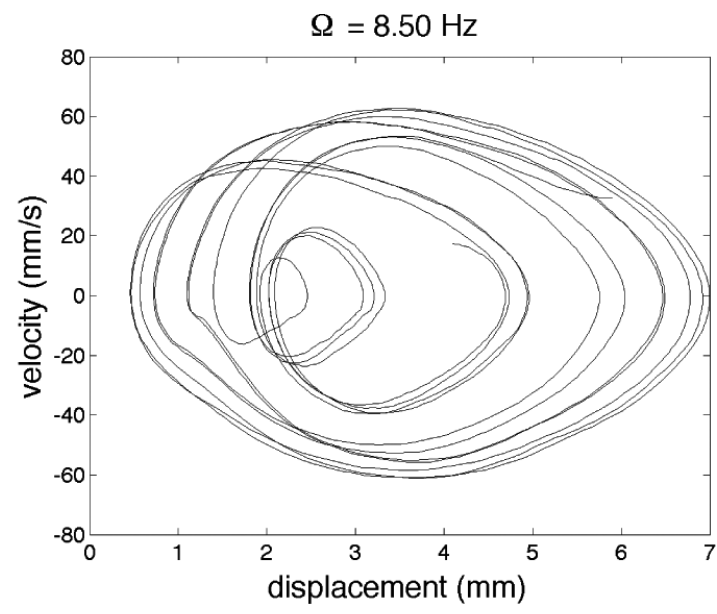

(b)

Fig. 9. Gap 0.0 mm. Nonperiodic behavior. Excitation frequency $8.50 \mathrm{~Hz}$ : a) Displacements; b) Hammer phase plane.

Therefore, the impact force ratio chart $\left(F_{i} / m g\right)$ in the frequency domain for this imposed gap is shown in Fig. 11(a). Although the maximum impact force on frequency band $z=1 / 2$ is higher than frequency band $z=$ $1 / 1$, is important to remember that in frequency $z=1 / 2$ impacts occur every two cycles and also the exctitation force inserted into the system increases with the square of the excitation frequency. Because the cart displacement is prescribed, as follows: 

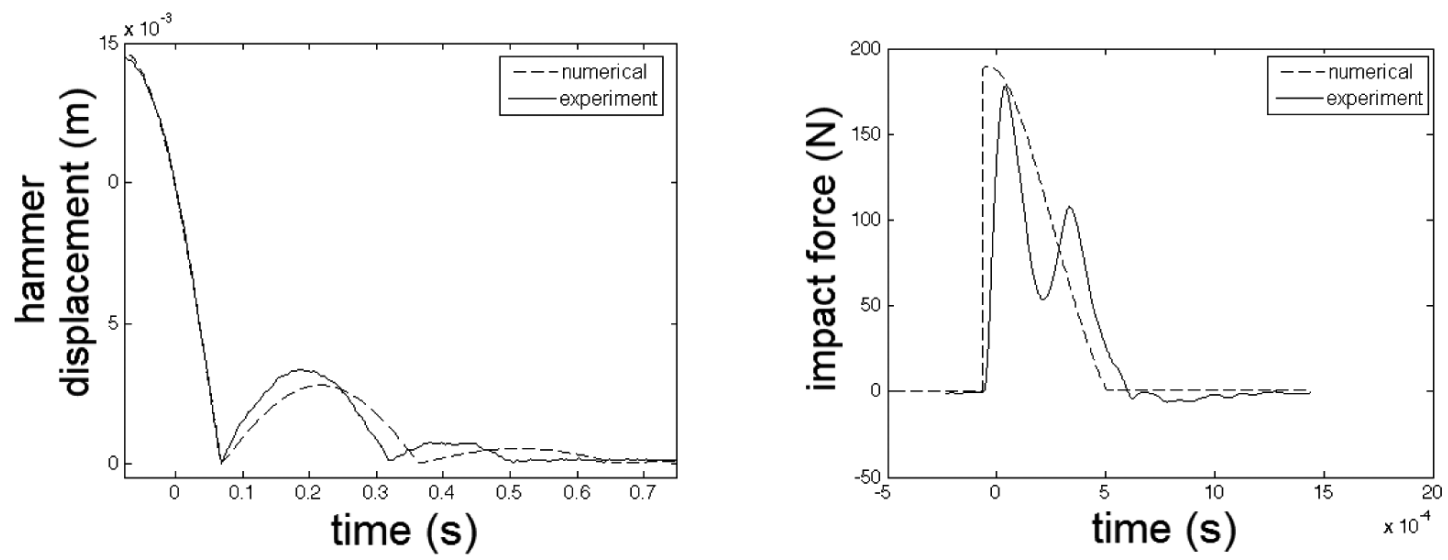

Fig. 10. Impact force parameters identification, spring-dashpot model. Comparison between experimental data and numerical simulation.

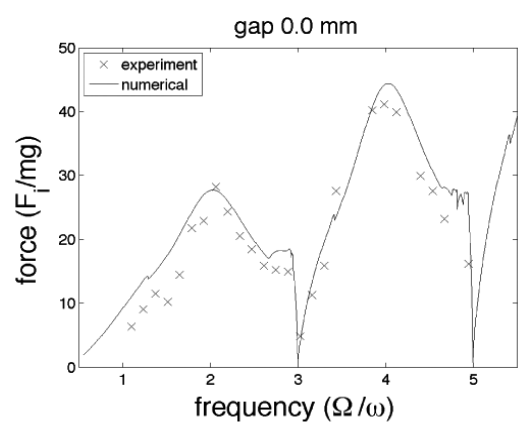

(a)

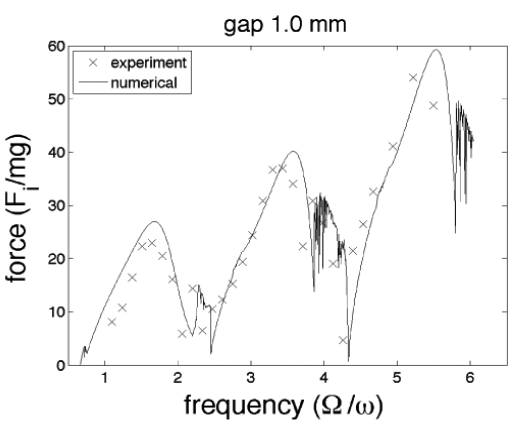

(b)

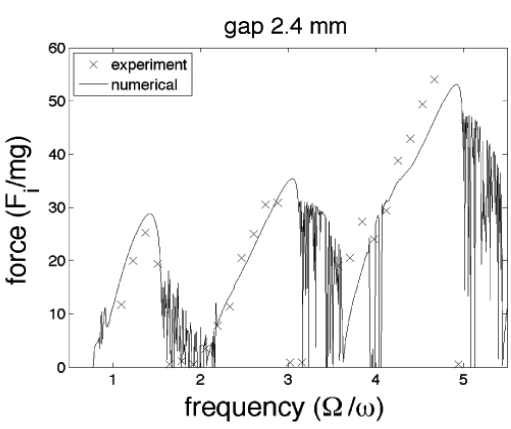

(c)

Fig. 11. Comparison between numerical simulation and experimental results. Non dimensional force versus non dimensional frequency. a) Gap $0.0 \mathrm{~mm}$; b) Gap $1.0 \mathrm{~mm}$; c) Gap $2.4 \mathrm{~mm}$.

$$
x_{\mathrm{exc}}=A_{0} \sin (\Omega t),
$$

it becomes that the magnitude of the excitation force $F_{\text {exc }}$ is, according to Newton's second law:

$$
F_{\text {exc }}=m_{\text {tot }} A_{0} \Omega^{2},
$$

where $m_{\text {tot }}$ is the total mass (cart and hammer combined), $A_{0}$ is the displacement amplitude of the cart and $\Omega$ is the excitation frequency.

Using a concept from the linear theory to describe a nonlinear behavior, the excitation frequency where the maximum impact force is achieved is defined as impact resonance. Since the hammer displacement is limited by a gap, an interesting phenomenon occurs. The occurrence of the impacts significantly changes the value of the impact resonance, as compared to the hammer resonance, as observed in Table 2. This change of resonance in the occurrence of impacts has already been studied [31] and these results were expected.

\subsection{Experimental results for gaps 1.0 and $2.4 \mathrm{~mm}$}

For these gap configurations the experimental results are similar to those observed for gap $0.0 \mathrm{~mm}$. However, the non-zero gap configurations show differences from the results for $0.0 \mathrm{~mm}$ gap. For instance, the occurrence of nonlinear jump was observed after the impact resonance for the $2.4 \mathrm{~mm}$ gap configuration. Another difference includes the appearance of situations of non-impact, due to the non-zero gap (at higher frequencies, for example, where the amplitudes developed are smaller than the gap). The non-dimensional chart $F_{i} / m g$ versus the nondimensional excitation frequency $\Omega / \omega$ for the gaps 1.0 and $2.4 \mathrm{~mm}$ are shown in Figs 11(b) and 11(c) respectively. 
Table 3

Parameters identification

\begin{tabular}{lcc}
\hline Parameter & Value & Unit \\
\hline Natural frequency, $\omega$ & 1.82 & $\mathrm{~Hz}$ \\
Hammer mass, $m$ & 0.298 & $\mathrm{~kg}$ \\
Cart mass, $M$ & 5.38 & $\mathrm{~kg}$ \\
Wire length, $l$ & 75 & $\mathrm{~mm}$ \\
Excitation amplitude, $A_{0}$ & 0.89 & $\mathrm{~mm}$ \\
Impact parameters & & \\
(spring-dashpot model) & & \\
Impact stiffness, $k_{i}$ & $5.5 \cdot 10^{6}$ & $\mathrm{~N} / \mathrm{m}$ \\
Impact damping, $c_{i}$ & $1.2 \cdot 10^{3}$ & $\mathrm{Ns} / \mathrm{m}$ \\
\hline
\end{tabular}

\subsection{Final remarks on the experimental data}

At this point the charts showing the behavior of the impact force over the excitation for each gap are compared. According to the charts shown in Fig. 11, in order to maximize the impact force, the hammer configuration with $0.0 \mathrm{~mm}$ gap presents higher impact forces in both regions of stable behavior $(z=1 / 1$ and $z=1 / 2)$. In addition, the $0.0 \mathrm{~mm}$ gap configuration shows no occurrence of nonlinear jump [32] after the maximum impact force in each frequency band. As mentioned, a variation of impact resonance frequency is observed as the gap varies, see Table 2 , as expected [31].

\section{Mathematical modeling and validation}

Using the Lagrange equation [30], with $\theta$ as the generalized coordinate, see Fig. 2(b), the hammer can be modeled as a single pendulum embedded in a cart with prescribed movement.

For the situation of no impact, i.e., $l \sin \theta-$ gap $>0$, considering that pendulum length is longer than impact gap, equation of motion is

$$
m l^{2} \ddot{\theta}-m l A_{0} \Omega^{2} \cos \theta \sin \Omega t+m g l \sin \theta=0 .
$$

The impact model used is the spring-dashpot model [33]. Figure 10 shows one comparison between experimental data and numerical simulation for the case where the cart is not moving and the hammer is released from a known initial condition. This result was used to identify the impact parameters.

Although the spring-dashpot model is not capable of reproducing the real impact force profile over time due to the jump caused by the damping force, this model generated satisfactory results. Impact parameters for this model are listed in Table 3.

Therefore, when the hammer is impacting the cart $(l \sin \theta-$ gap $\leqslant 0)$, the equation of motion will change to

$$
m l^{2} \ddot{\theta}-m l A_{0} \Omega^{2} \cos \theta \sin \Omega t+m g l \sin \theta=-F_{i} l ; F_{i}=k_{i} \delta+c_{i} \dot{\delta},
$$

where the penetration $\delta$ and the velocity of penetration, $\dot{\delta}$ are described as

$$
\begin{aligned}
& \delta=l \sin \theta-\text { gap }, \\
& \dot{\delta}=l \dot{\theta} \cos \theta .
\end{aligned}
$$

It is important to emphasize that the generalized coordinate $\theta$ (and therefore $\dot{\theta}$ ) is embedded in the cart. To compare the numerical results with the experimental data, where the linear displacement is measured outside the cart, the following transformations must take place:

$$
\begin{aligned}
& x=l \sin \theta+A_{0} \sin (\Omega t) \\
& \dot{x}=l \dot{\theta} \cos \theta+A_{0} \Omega \cos (\Omega t)
\end{aligned}
$$

The system without impact presents some degree of damping. However, the mathematical modeling does not take this into consideration. For the system without impact, the test rig parameters are identified and the hammer natural frequency is obtained. These results are shown in Table 3. 
According to the Filippov theory [34-36], the mathematical modeling presented is described by a differential equation with a discontinuous right-hand side. Therefore, the state space $\dot{\mathbf{x}}=f(\mathbf{x}), \mathbf{x} \in \mathbf{R}^{n}$ may be split into two subspaces $\Gamma_{-}$and $\Gamma_{+}$, separated by a hyper-surface $\Sigma$. Hyper-surface is defined by a scalar function $h(\mathbf{x})$. Consequently, the state space $\mathbf{x}$ is in $\Sigma$ when $h(\mathbf{x})=0$. Hence, it is possible to define the subspaces $\Gamma_{-}$and $\Gamma_{+}$, as well as the hyper-surface $\Sigma$, using the sets:

$$
\begin{aligned}
\Gamma_{-} & =\left\{\mathbf{x} \in \mathbf{R}^{n} \mid h(\mathbf{x})<0\right\} \\
\Sigma & =\left\{\mathbf{x} \in \mathbf{R}^{n} \mid h(\mathbf{x})=0\right\} \\
\Gamma_{+} & =\left\{\mathbf{x} \in \mathbf{R}^{n} \mid h(\mathbf{x})>0\right\}
\end{aligned}
$$

Some physical systems need different interfaces in order to perform a correct description of the transitions. The impact force model used in the mathematical modeling is an example. Due to the nature of the impact model, the contact between the mass and the support occurs whenever the linear displacement becomes equal to the contact gap. However, the mass loses contact with the support when the contact force vanishes. Two indicator functions are used to define the system subspaces. More details on this mathematical procedure can be found in the works of Divenyi et al. [23,24].

$$
\begin{aligned}
& h_{\alpha}(\theta, \dot{\theta})=l \sin \theta-g a p \\
& h_{\beta}(\theta, \dot{\theta})=k_{i} \delta+c_{i} \dot{\delta},
\end{aligned}
$$

where the penetration $\delta$ and velocity of penetration $\dot{\delta}$ are already defined in Eq. (5).

The mass is not in contact with the support if the state vector $\mathbf{x}=(\theta, \dot{\theta}) \in \Gamma_{-}$, in other words:

$$
\Gamma_{-}=\left\{\mathbf{x} \in \mathbf{R}^{2} \mid h_{\alpha}(\theta, \dot{\theta})<0 \text { or } h_{\beta}(\theta, \dot{\theta})<0\right\} .
$$

For the case when there is contact between the mass and the support:

$$
\Gamma_{+}=\left\{\mathbf{x} \in \mathbf{R}^{2} \mid h_{\alpha}(\theta, \dot{\theta})>0 \text { and } h_{\beta}(\theta, \dot{\theta})>0\right\} .
$$

The hyper-surface $\Sigma$ consists of the conjunction of two surfaces, $\Sigma_{\alpha}$ and $\Sigma_{\beta}$. The hyper-surface $\Sigma_{\alpha}$ defines the transition from $\Gamma_{-}$to $\Gamma_{+}$, i.e., when the mass initiates the contact with the support $(l \sin \theta-g a p=0)$,

$$
\Sigma_{\alpha}=\left\{\mathbf{x} \in \mathbf{R}^{2} \mid h_{\alpha}(\theta, \dot{\theta})=0 \text { and } h_{\beta}(\theta, \dot{\theta}) \geqslant 0\right\} .
$$

Surface $\Sigma_{\beta}$ defines the transition from $\Gamma_{+}$to $\Gamma_{-}$as the contact is lost when the impact force vanishes:

$$
\Sigma_{\beta}=\left\{\mathbf{x} \in \mathbf{R}^{2} \mid h_{\alpha}(\theta, \dot{\theta}) \geqslant 0 \text { and } h_{\beta}(\theta, \dot{\theta})=0\right\} .
$$

Consequently, the state equation of this discontinuous system is written as follows:

$$
\dot{\mathbf{x}}=f(\mathbf{x}, t)=\left\{\begin{array}{l}
f_{-}(\mathbf{x}, t), \mathbf{x} \in \Gamma_{-} \\
\overline{c o}\left\{f_{-}(\mathbf{x}, t), f_{+}(\mathbf{x}, t)\right\}, \mathbf{x} \in \Sigma \\
f_{+}(\mathbf{x}, t), \mathbf{x} \in \Gamma_{+}
\end{array}\right.
$$

where

$$
\begin{aligned}
& f_{-}(\mathbf{x}, t)=\left[\begin{array}{c}
\dot{\theta} \\
\frac{A_{0} \Omega^{2}}{l} \cos \theta \sin \Omega t-\frac{g}{l} \sin \theta
\end{array}\right] ; \mathbf{x} \in \Gamma_{-} \\
& f_{+}(\mathbf{x}, t)=\left[\begin{array}{c}
\dot{\theta} \\
\frac{A_{0} \Omega^{2}}{l} \cos \theta \sin \Omega t-\frac{g}{l} \sin \theta-\frac{1}{m l}\left(k_{i} \delta+c_{i} \dot{\delta}\right)
\end{array}\right] ; \mathbf{x} \in \Gamma_{+} \\
& \overline{c o}\left\{f_{-}(\mathbf{x}, t), f_{+}(\mathbf{x}, t)\right\}=\left[\begin{array}{c}
\dot{\theta} \\
\frac{A_{0} \Omega^{2}}{l} \cos \theta \sin \Omega t-\frac{g}{l} \sin \theta-\frac{1}{m l}\left(c_{i} \dot{\delta}\right)
\end{array}\right] ; \text { in } \Sigma_{\alpha}
\end{aligned}
$$




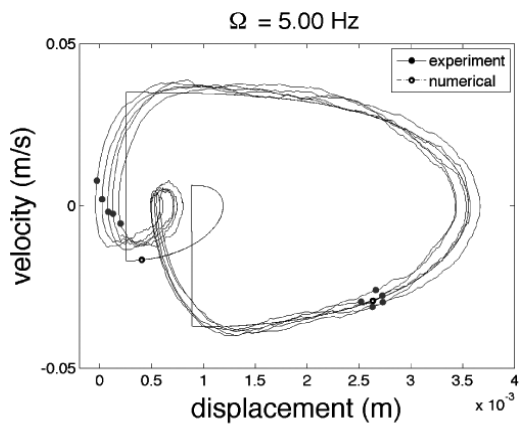

(a)

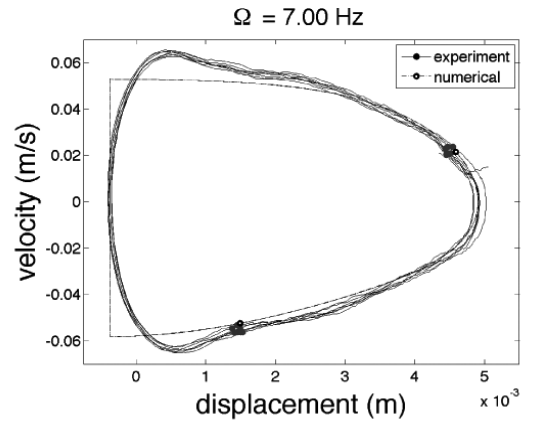

(b)

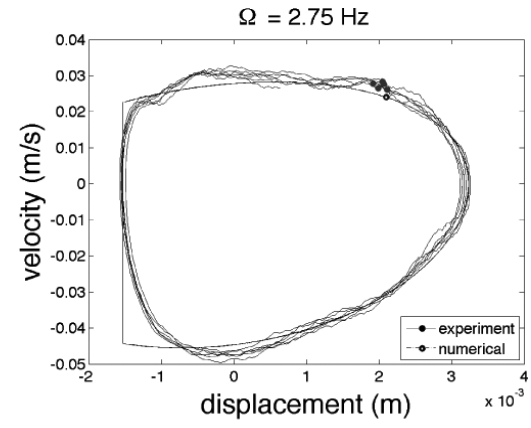

(c)

Fig. 12. Phase plane and Poincaré maps, comparison between numerical simulation (blue) and experimental results $($ red): a) Gap 0.0 mm, $\Omega / \omega=$ 2.7; b) Gap $0.0 \mathrm{~mm}, \Omega / \omega=3.8$; c) Gap $1.0 \mathrm{~mm}, \Omega / \omega=1.5$.

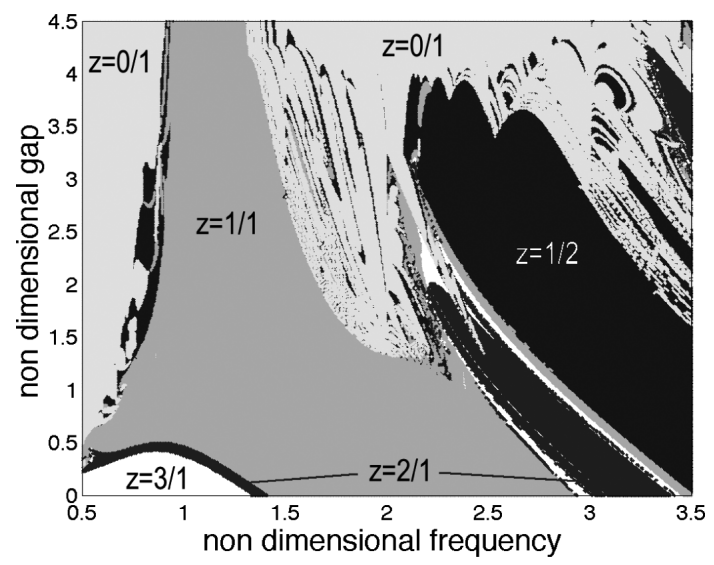

Fig. 13. Regions of stable periodic behavior.

$$
\overline{c o}\left\{f_{-}(\mathbf{x}, t), f_{+}(\mathbf{x}, t)\right\}=\left[\begin{array}{c}
\dot{\theta} \\
\frac{A_{0} \Omega^{2}}{l} \cos \theta \sin \Omega t-\frac{g}{l} \sin \theta
\end{array}\right] ; \text { in } \Sigma_{\beta} .
$$

This approach allows one to numerically integrate non-smooth systems [23].

The comparison between numerical simulation and experimental data starts with the chart of the non-dimensional force $\left(F_{i} / m g\right)$ in the non-dimensional frequency domain $(\Omega / \omega)$, for each gap imposed on the test rig. These results are shown in Fig. 11.

Figure 12 shows the phase plane and Poincaré map of the hammer, comparing the experimental data with simulation results. Charts show good agreement, especially within every frequency band, showing satisfactory agreement after bifurcation.

Although the impact force charts shown in Fig. 11 give some important information regarding the impact force amplitude and the impact resonance, such charts provide neither information about the characteristics of the impact force, nor details on the transition between frequency bands. To better visualize the behavior of this dynamical system, two nonlinear tools are used. The tool shows a map of regions of stable impact behavior, called by the authors Peterka map [12,27,25,26], shown in Fig. 13, which provides information about the characteristic of the impact force as the gap is varied and the range of excitation frequencies is covered. The map is generated by analysing the system behavior in time domain for each set of controlled parameters (excitation frequency and impact gap). From this chart one can see the areas where the two frequencies bands occur, as noted by the red $(z=1 / 1)$ and green $(z=$ $1 / 2$ ) areas. The second tool is a bifurcation diagram [32], where the bifurcation in the $z=1 / 1$ behavior is observed in more detail, see Fig. 15. 


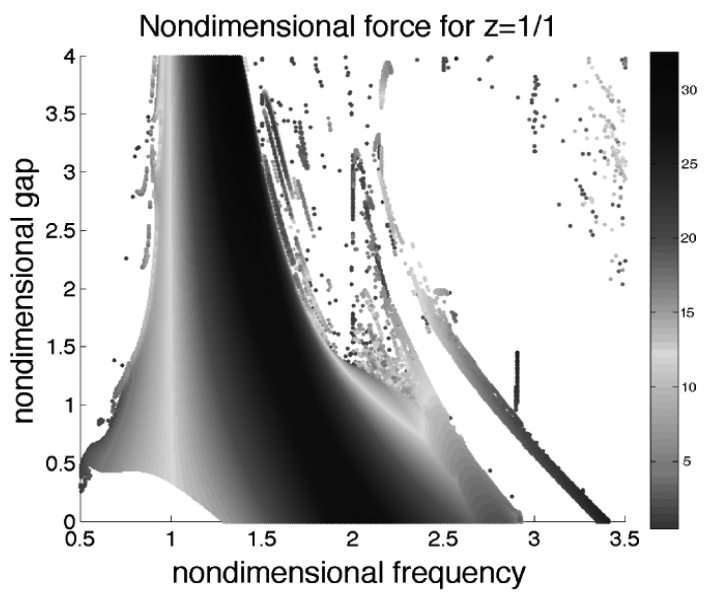

(a)

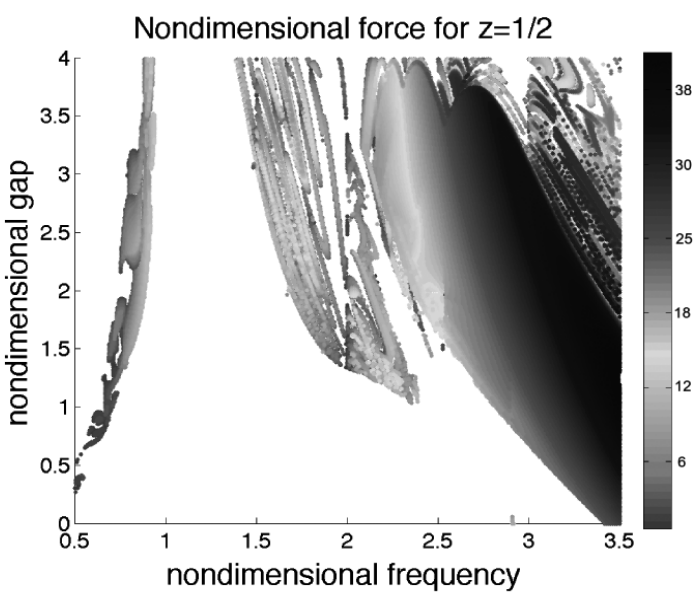

(b)

Fig. 14. a) Regions of stable periodic behavior with impact force magnitude addressed, $z=1 / 1$ behavior; b) Regions of stable periodic behavior with impact force magnitude addressed, $z=1 / 2$ behavior.

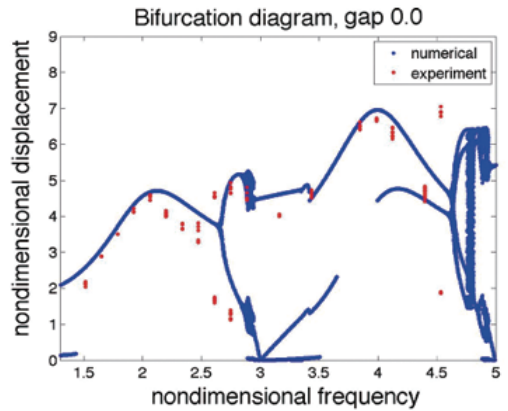

(a)

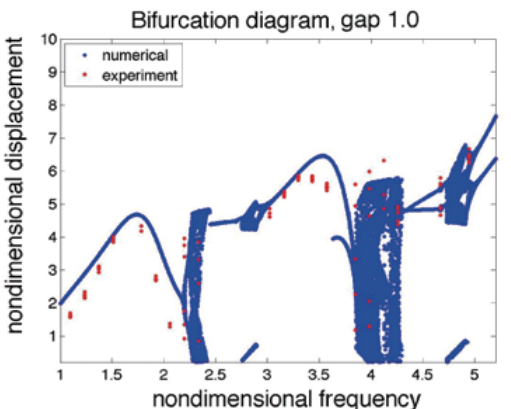

(b)

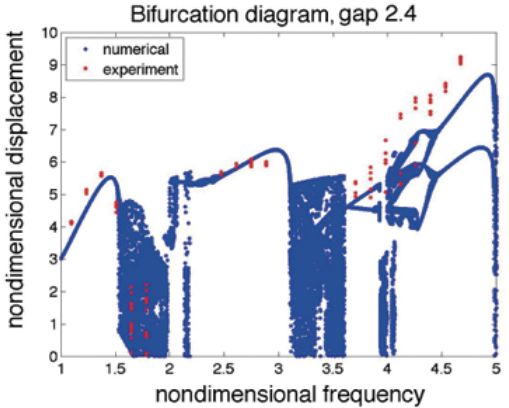

(c)

Fig. 15. Bifurcation diagram: hammer displacement, numerical simulation (blue)/ experimental data (red) comparison: a) Gap 0.0 mm; b) $1.0 \mathrm{~mm}$; c) $2.4 \mathrm{~mm}$.

Although the Peterka map provides important information about the condition of impact, no information regarding the impact magnitude is given. To overcome this problem, a slight variance of the Peterka map is suggested. The relevant impact condition is $z=1 / 1$. Therefore, just this area in the Peterka map is addressed. For each gap/ frequency combination, the impact force magnitude is obtained and plotted in colors, see Fig. 14(a). Such methodology is also applied to impact behavior $z=1 / 2$, as shown in Fig. 14(b).

Chart shown in Fig. 14(a) provides several important facts about the system behavior and it confirms some aspects observed during the experimental analysis. First, it confirms that the impact force when the hammer is excited in its natural frequency generates impact forces that are 3 times smaller in magnitude than the maximum force. It also shows that the maximum impact force for each given gap does not occur at the $z=1 / 1$ boundary, except for high values of gap. Finally, the chart confirms the recommendation to operate in the field using the $0.0 \mathrm{~mm}$ gap, because the magnitude of the impact force is in the same value as the impact force in higher gap values. In addition, non-zero gap values are known to present nonlinear jumps.

\section{Conclusions}

The purpose of this work is the experimental investigation and the mathematical modeling of the impact force behavior in a vibro-impact system, where an impact pendulum is mounted on a cart that moves with a prescribed 
displacement. The dynamics of the system was evaluated considering different excitation frequencies and changing the impact gap. Experimental results show that even with a complex hammer dynamics, the system can be modeled as a single-degree-of-freedom embedded pendulum, where such results are widely available in the literature. Although the literature shows several studies dealing with vibro-impact systems, as listed, there is little concern regarding the force magnitude developed by the impacts. Since the ulterior motivation relies on a hybrid technique to drill hard rock formations, special attention is dedicated in this work regarding this issue, as well as which system parameters combination develops higher impact forces.

Previous authors' work using an elastic supported hammer presented an interesting amount of different nonlinearities [28], but included discrepancies in the validation due to hammer dynamics. This lead to the present paper where the experiment consists of a simpler hammer set up. The comparison between the numerical simulation and the experimental data was satisfactory, showing the capability of the mathematical model to predict the maximum impact force and the impact resonance for all gaps imposed.

Also, a new methodology is used to better visualize each impact force behavior in the map of regions of stable behavior [29], plotting one impact force characteristic at a time and adding colors to the third coordinate $F_{i}$. This methodology provided important information regarding the hammer behavior and confirmed some aspects observed during the experimental analysis.

Finally, in this hammer set up, in order to maximize the impact force, the hammer configuration with $0.0 \mathrm{~mm}$ gap presents higher impact forces in both regions of stable behavior $(z=1 / 1$ and $z=1 / 2)$.

\section{Acknowledgments}

The authors wish to thank CNPq and FAPERJ for its support of this research and Dr. Luiz Fernando Franca for his helpful discussions and suggestions.

\section{References}

[1] S. Chen, Linear and Nonlinear Dynamics of Drillstrings, PhD thesis, Faculté des Sciences Appliquées, Université de Liège, Liège, Belgium (1995).

[2] D.W. Dareing, F.H. Deily, G.H. Paff, J.E. Ortloff and R.D. Lynn, Downhole Measurements of Drill String Forces and Motions, ASME Journal of Engineering for Industry (May 1968), 217-225.

[3] M.W. Dykstra, Nonlinear Drillstring Dynamics, PhD thesis, Department of Petroleum Engineering, University of Tulsa, Oklahoma, USA, 1996.

[4] R.A. Cunningham, Analysis of Downhole Measurements of Drill String Forces and Motions, ASME Journal of Engineering for Industry (May 1968), 208-216.

[5] A.D. Batako, V.I. Babitsky and N.A. Halliwell, A self-excited system for percussive-rotary drilling, Journal of Sound and Vibration 259 (2003), 97-118.

[6] A.D. Batako, V.I. Babitsky and N.A. Halliwell, Modelling of vibro-impact penetration of self-exciting percussive-rotary drill bit, Journal of Sound and Vibration 271 (2004), 209-225.

[7] L.F.P. Franca and H.I. Weber, Experimental and Numerical Study of a new Resonance Hammer Drilling Model with Drift, Chaos, Solitons and Fractals 21 (2004), 789-801.

[8] M. Wiercigroch, R.D. Neilson and M.A. Player, Material removal rate prediction for ultrasonic drilling of hard materials using an impact oscillator approach, Physics Letters A 259, (1999), 91-96.

[9] M. Wiercigroch, J. Wojewodab and A.M. Krivtsov, Dynamics of ultrasonic percussive drilling of hard rocks, Journal of Sound and Vibration 280 (2005), 739-757.

[10] V.I. Babitsky, A.N. Kalashnikovb, A. Meadowsa and A.A.H.P. Wijesundara, Ultrasonically assisted turning of aviation materials, Journal of Materials Processing Technology 132 (2003), 157-167.

[11] K.R. Asfar and S.N. Akour, Optimization Analysis of Impact Viscous Damper for Controlling Self-Excited Vibration, Journal of Vibration and Control 11(1) (2005), 103-120.

[12] F. Peterka, More detail view on the dynamics of the impact damper, Facta Universitatis Series: Mechanics, Automatic Control and Robotics 3(14) (2003), 907-920.

[13] V.I. Babitsky, Hand-Held Percussion Machine As Discrete Non-Linear Converter, Journal of Sound and Vibration 214 (1998), $165-182$.

[14] S.W. Shaw and P.J. Holmes, Periodically forced linear oscillator with impacts: chaos and long-period motions, Physical Review Letters 51 (1983), 623-626

[15] S.W. Shaw, Forced vibrations of a beam with one-sided amplitude constraint: theory and experiment, Journal of Sound and Vibration 99 (1985), 199-212. 
[16] J.M.T. Thompson, A.R. Bokaian and R. Ghaffari, Subharmonic resonances and chaotic motions of a bilinear oscillator, Journal of Applied Mathematics 31 (1983), 207-234.

[17] J. Ing, E. Pavlovskaia, M. Wiercigroch and S. Banerjee, Experimental study of impact oscillator with one-sided elastic constraint, Philosophical Transactions of the Royal Society A 366 (2008), 679-704.

[18] B.P. Mann, R.E. Carter and S.S. Hazra, Experimental study of an impact oscillator with viscoelastic and Hertzian contact, Nonlinear Dynamics $\mathbf{5 0}$ (2007), 587-596.

[19] N. Hinrichs, M. Oestreich and K. Popp, Dynamics of oscillators with impact and friction, Chaos, Soliton and Fractals 8 (1997), $535-558$.

[20] M.D. Todd and L.N. Virgin, An experimental impact oscillator, Chaos, Solitons and Fractals 8 (1997), 699-715.

[21] P.T. Piiroinen, L.N. Virgin and A.R. Champneys, Chaos and period adding; experimental and numerical verification of the grazing bifurcation, Journal of Nonlinear Science 14 (2004), 383-404.

[22] V.I. Babitsky and V.L. Krupenin, Vibration of Strongly Nonlinear Discontinuous Systems, Springer 2001.

[23] S. Divenyi, M.A. Savi, L.F.P. Franca and H.I. Weber, Nonlinear Dynamics and Chaos in Systems with Discontinuous Support, Shock and Vibration 13 (2006), 315-326.

[24] S. Divenyi, M.A. Savi, L.F.P. Franca and H.I. Weber, Numerical and Experimental Investigations of the Nonlinear Dynamics and Chaos in Non-Smooth Systems, Journal of Sound and Vibration 301 (2007), 59-73.

[25] F. Peterka, Bifurcations and Transition Phenomena in an Impact Oscillator, Chaos, Solitons and Fractals 7(10) (1996), $1635-1647$.

[26] F. Peterka and B. Blazejczyk-Okolewska, An investigation of the dynamic system with impacts, Chaos, Solitons and Fractals 9(8) (1998), 1321-1338.

[27] F. Peterka, T. Kotera and S. Cipera, Explanation of appearance and characteristics of intermittency chaos of the impact oscillator, Chaos, Solitons and Fractals 19 (2004), 1251-1259.

[28] R.R. Aguiar and H.I. Weber, Mathematical modeling and experimental investigation of an embedded vibro-impact system, Nonlinear Dynamics 65 (2011), 317-334.

[29] R.R. Aguiar, Experimental investigation and numerical analysis of the vibro-impact phenomenon, DSc thesis, Departamento de Engenharia Mecânica, PUC-Rio, Rio de Janeiro, Brazil (2010).

[30] D.J. Inman, Engineering Vibration, Prentice Hall Inc., USA, 1996.

[31] M.C. Mattos and H.I. Weber, Some Interesting Characteristics of a Simple Autonomous Impact System with Symmetric Clearance, ASME - Design Engineering Conference, 5 pgs, CD-ROM (1997).

[32] S.H. Strogatz, Nonlinear Dynamics and Chaos, Westview Press, USA, 2000

[33] G. Gilardi and I. Sharf, Literature survey of contact dynamics modelling, Mechanism and Machine Theory 37 (2002), $1213-1239$.

[34] R.I. Leine, Bifurcations in discontinuous mechanical systems of Filippov-type, PhD thesis, Technische Universiteit Eindhoven, 2000.

[35] R.I. Leine, D.H. van Campen and B.L. van de Vrande, Bifurcations in nonlinear discontinuous systems, Nonlinear Dynamics 23(2) (2000), 105-164.

[36] R.I. Leine and H. Nijmeijer, Dynamics and Bifurcations of Non-Smooth Mechanical Systems, Springer, 2004. 

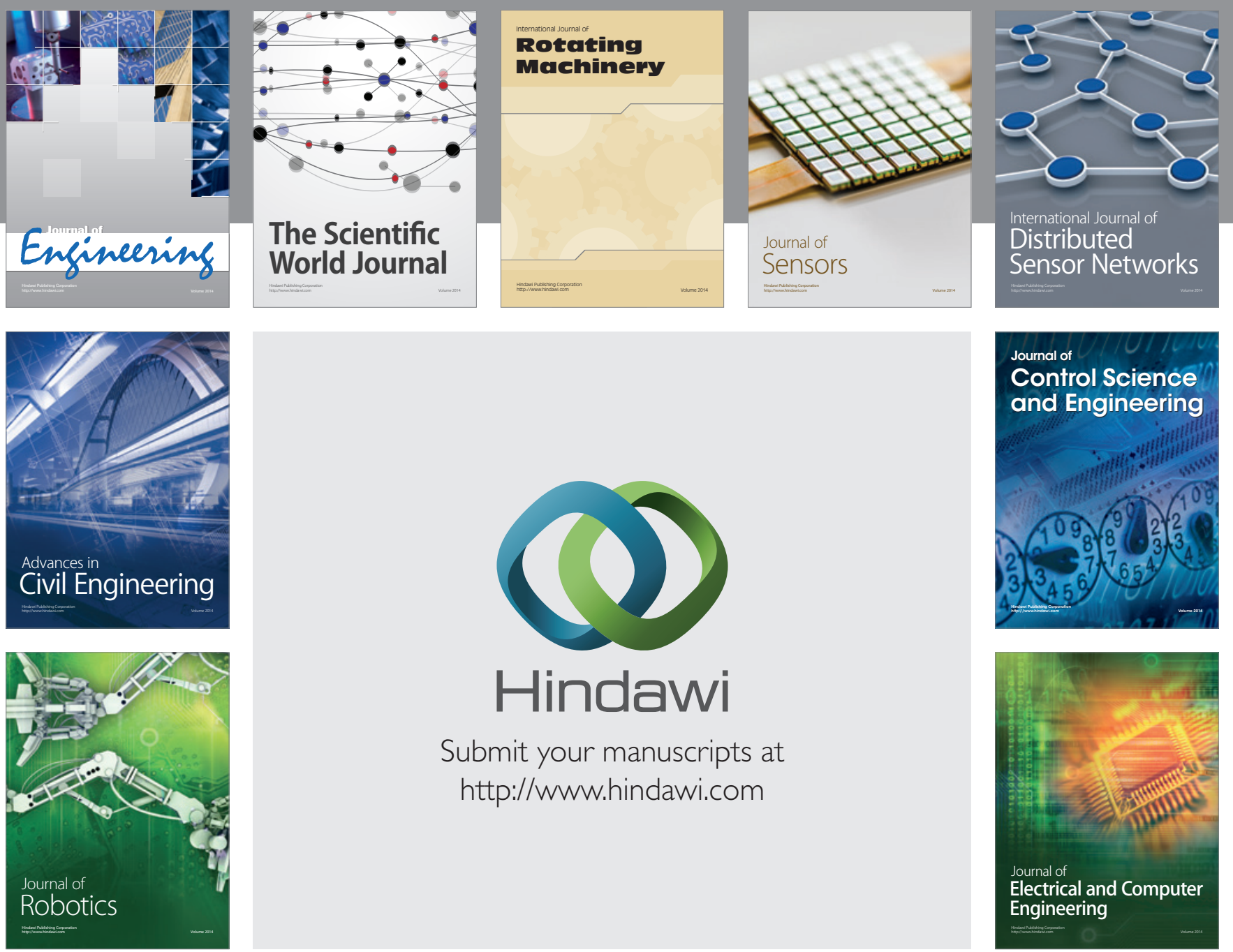

Submit your manuscripts at

http://www.hindawi.com
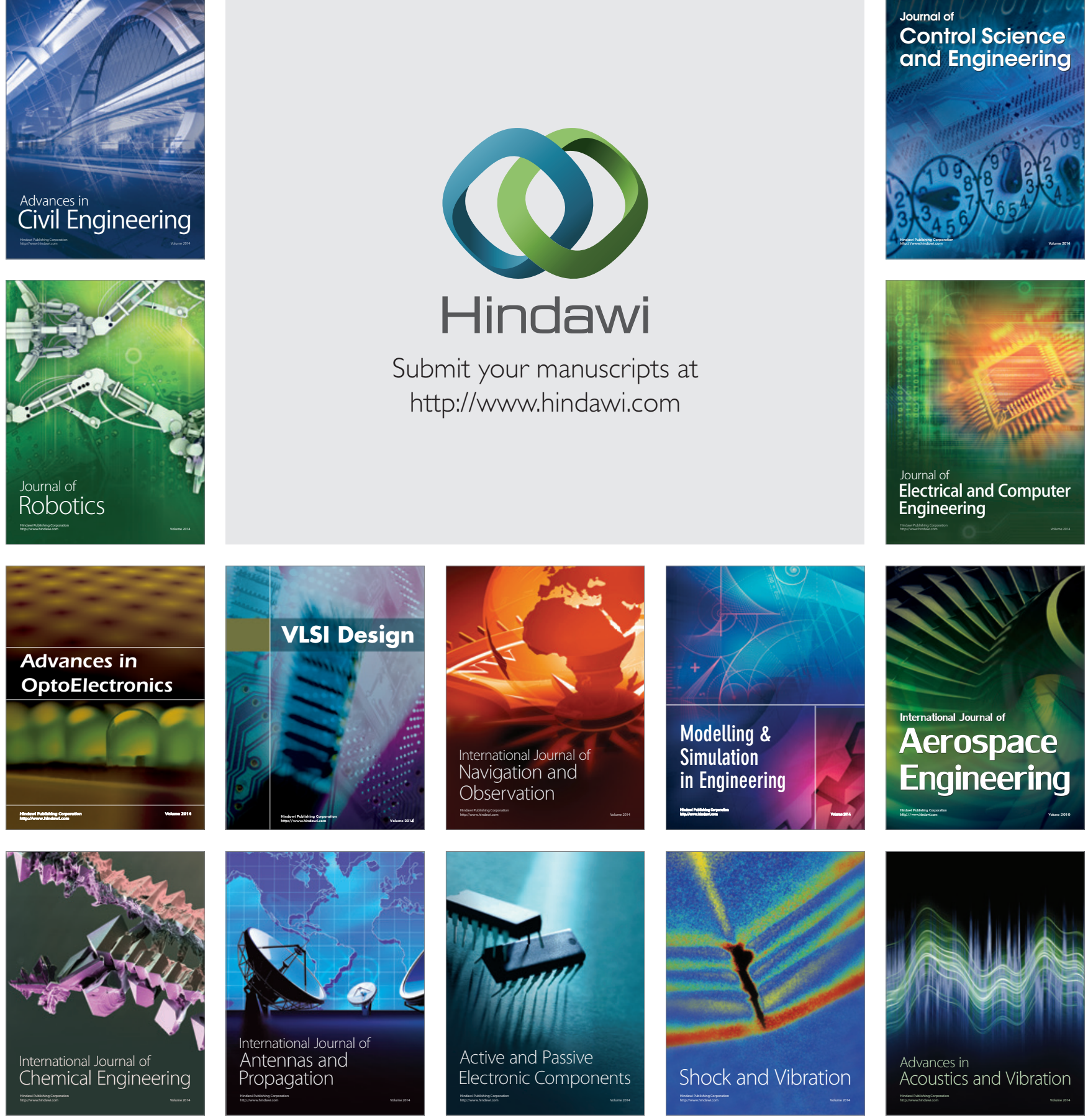